\title{
The effect of centromere protein $U$ silencing by lentiviral mediated RNA interference on the proliferation and apoptosis of breast cancer
}

\author{
SHUANG-YAN LIN ${ }^{1 *}$, YAN-BO LV ${ }^{1 *}$, GEN-XIANG MAO ${ }^{2}$, XU-JIAO CHEN $^{3}$ and FANG PENG ${ }^{1}$ \\ ${ }^{1}$ Department of Pathology; ${ }^{2}$ Department of Geriatrics, Zhejiang Provincial Key Lab of Geriatrics; \\ ${ }^{3}$ Department of Geriatrics, Zhejiang Hospital, Hangzhou, Zhejiang 310013, P.R. China
}

Received March 16, 2017; Accepted August 14, 2018

DOI: $10.3892 / \mathrm{ol} .2018 .9477$

\begin{abstract}
Centromere protein U (CENPU) is a novel transcriptional repressor that is associated with different types of cancer. However, its function in breast cancer is poorly understood. In the present study, it was identified that CENPU was highly expressed in breast cancer tissues compared with expression in normal breast tissues $(\mathrm{P}=0.001)$. Furthermore, the $C E N P U$ mRNA level in tumors was often elevated, compared with the matched adjacent normal breast cancer tissue specimens in the dataset from The Cancer Genome Atlas database $(n=106$; $\mathrm{P}<0.001)$. To understand the function of CENPU in human breast carcinogenesis, its effects on the proliferation, apoptosis and cell cycle progression of MDA-MB-231 cells were examined using the lentiviral-mediated $C E N P U$ knockdown approach. The RNA and protein expression levels in the transfected cells were monitored using reverse transcription-quantitative polymerase chain reaction and western blotting, respectively. The mRNA and protein expression levels of the CENPU gene were significantly lower in the CENPU-shRNA transfected cells than in the control $(\mathrm{P}<0.01)$, indicating successful gene expression knockdown. Post-transfection, cell counting and MTT analysis revealed that the proliferation activity was significantly suppressed in CENPU knockdown cells relative to the control $(\mathrm{P}<0.01)$. Additionally, fluorescence activated cell sorting analysis revealed that the $(\mathrm{G} 2+\mathrm{S})$ phase fraction was significantly declined in $C E N P U$ knockdown cells relative to the control; while the G1 phase fraction was significantly increased $(\mathrm{P}<0.01)$ and the percentage of the apoptotic cells was significantly increased $(\mathrm{P}<0.01)$. In conclusion,
\end{abstract}

Correspondence to: Professor Fang Peng, Department of Pathology, Zhejiang Hospital, 12 Lingyin Road, Hangzhou, Zhejiang 310013, P.R. China

E-mail: pengfang999@139.com

\section{${ }^{*}$ Contributed equally}

Key words: breast cancer, centromere protein U, proliferation, cell cycle, apoptosis downregulation of CENPU gene expression may inhibit cell proliferation and cell cycle progression, and increase the apoptosis of the breast cancer cells. These results suggested a possible function of this protein in breast cancer pathogenesis and prognosis.

\section{Introduction}

Breast cancer is one of the most common human neoplasms, accounting for $\sim 1 / 4$ of cases of cancer in females worldwide and $27 \%$ of cancer cases in developed countries (1). Breast cancer can also occur in men; however, it is more common in women (2). It is the leading cause of mortality in women with a global incidence of $>1,000,000$ cases annually (3). It typically has a poor prognosis due to due to the majorty of diagnoses ocuring at an advanced stage of disease (4). As a complex disease, alteration of cellular signals serves important functions in breast cancer development. In order to reduce the mortality rate of breast cancer, molecular-targeted therapy is emerging as an alternative treatment (5). In previous decades, efforts have been concentrated on the discovery of molecular targets; numerous molecular targeted therapies approved by the Food and Drug Administration (FDA), have demonstrated remarkable clinical success in the treatment of breast cancer (6). However, breast cancer associated incidence and mortality has been steadily increasing from 2000 through to $2011(7,8)$. Studies to identify molecular targets that may improve therapeutic strategies and clinical outcomes are required.

Centromere protein $\mathrm{U}(C E N P U)$ encodes for a putative transcriptional repressor whose deregulation has been associated with the development of several types of cancer, including glioblastoma, and prostate and luminal breast cancer (9-12). It is localized to human chromosome $4 \mathrm{q} 35$.1 and is expressed in the nuclei and cytoplasm of the cells of a number of tissues, including fetal liver, bone marrow, thymus and testicular tissue (13). The function and mechanism of CENPU deregulation in breast cancer is poorly understood. In the present study, $C E N P U$ expression was examined in human breast cancer tissue and cell lines. Using the lentiviral mediated RNA interference approach; the effects of CENPU expression inhibition on the cell proliferation, apoptosis and cell cycle progression of breast cancer MDA-MB-231 cells were investigated. 


\section{Materials and methods}

Patients and tissue samples. Paired tumor and normal breast tissue specimens were obtained from 15 patients with breast cancer who were surgically treated at Zhejiang Hospital (Hangzhou, China) between January 2016 and December 2017. Written informed consent was obtained from each of the patients, in compliance with the Declaration of Helsinki, and the study was approved by the Medical Ethics Committee of Zhejiang Hospital (approval no. 2017-19K). The normal breast tissues were removed a distance of $>3 \mathrm{~cm}$ from the tumor margin. All specimens were fixed in $10 \%$ neutral formalin at room temperature for 24-36 $\mathrm{h}$ prior to analysis.

Immunohistochemicalanalysis. All tissues were formalin-fixed and paraffin-embedded. Tissues were sectioned into 4- $\mu \mathrm{m}$ slices. Then the sections were deparaffinized in xylene and rehydrated in a descending ethylalcohol series $(100,95,85$ and $75 \%$ ) followed by distilled water. Immunohistochemical staining was performed using an enhanced labeled polymer method (14). In brief, tissue sections were subjected to a boiling antigen retrieval procedure in Ethylene Glycol Tetraacetic Acid buffer (EGTA, pH 9.0) for 25 min. Following blocking of endogenous peroxidase activity with $3 \%$ hydrogen peroxide (Zhongshan Golden Bridge Biotechnology Co., Ltd., Beijing, China) at room temperature for $10 \mathrm{~min}$, the sections were incubated overnight at $4^{\circ} \mathrm{C}$ with a polyclonal rabbit anti-human CENPU antibody (dilution, 1:50; cat. no. ab117078; Abcam, Cambridge, UK). Negative control sections were treated with PBS (Zhongshan Golden Bridge Biotechnology Co., Ltd) instead of the primary antibody. Following incubation with the EnVision System-Horseradish Peroxidase (PV-8000, Zhongshan Golden Bridge Biotechnology Co., Ltd) at $37^{\circ} \mathrm{C}$ for $20 \mathrm{~min}$, the reaction products were visualized by $3,3^{\prime}$-diaminobenzidine staining (Zhongshan Golden Bridge Biotechnology Co. Ltd) and counterstaining with hematoxylin at room temperature for $1 \mathrm{~min}$. Immunohistochemical scoring was analyzed by Carl Zeiss Microscope (at x400 and x200 magnification), and this was conducted by two pathologists by consensus without knowledge of the clinicopathological information. CENPU expression levels were scored semi-quantitatively by assessing the intensity of staining $(0$, no staining; 1 , mild staining; 2 , moderate staining; and 3, strong staining) and by the percentage of positively stained cells $(0,<30 \% ; 1,30-49 \%$; $2,50-69 \%$; and $3, \geq 70 \%$ ). The index sum was the result of totaling the staining intensity and percentage scores. Based on the results of previous studies $(15,16)$, a specimen with a final score $>4$ was considered as positive, while the others were considered to be negative.

Cell culture. The normal breast MCF-10A cell line was purchased from the cell bank of GuanDao Biotechnology (http://shgdbio.company.lookchem.cn/; Shanghai, China), and the breast cancer MDA-MB-231 cell line was obtained from the American Type Culture Collection (Manassas, VA, USA) and the T-47D cell line was obtained from the cell bank of the Chinese Academy of Sciences (Shanghai, China). The cells were cultured and passaged in Dulbecco's modified Eagle's medium (DMEM) (Gibco; Thermo Fisher Scientific, Inc., Waltham, MA, USA) supplemented with $10 \%$ Fetal Bovine
Serum (FBS; Thermo Fisher Scientific, Inc.) at $37^{\circ} \mathrm{C}$ in a $95 \%$ relative humidified atmosphere containing $5 \% \mathrm{CO}_{2}$. The medium was changed every 2 days for all cell lines.

The Cancer Genome Atlas (TCGA) gene expression data analysis. Public data of CENPU gene expression for patients with breast cancer were obtained from the Cancer Genome Atlas Project (TCGA; http://cancergenome.nih.gov/) and the Firebrowse dataset (http://firebrowes.org/) (17). The matched tumor and non-tumor specimens were available for a total of 106 patients.

$R N A$ isolation and reverse transcription-quantitative polymerase chain reaction ( $R T-q P C R)$. RNA of all three cell lines was extracted using a TRIzol ${ }^{\circledR}$ kit (Thermo Fisher Scientific, Inc.), according to the manufacturer's protocol. RNA purity and concentration were assessed using a NanoDrop 2000c spectrophotometer (Thermo Fisher Scientific, Inc.). Reverse transcription was performed with the M-MLV RTase cDNA Synthesis kit (Promega Corporation, Madison, WI, USA). First, $2.0 \mu \mathrm{g}$ total RNA and $1 \mu \mathrm{l}$ Oligo dT $(0.5 \mu \mathrm{g} / \mu \mathrm{l}$; Shanghai Sangon, China) were added to a PCR vial, and then RNA-free $\mathrm{H}_{2} \mathrm{O}$ was added to reach a total vial volume of $10 \mu \mathrm{l}$. The vial was mixed gently, instantaneously centrifuged, placed in a $70^{\circ} \mathrm{C}$ warm bath for $10 \mathrm{~min}$ and then immediately transferred to an ice-water bath $\left(0^{\circ} \mathrm{C}\right)$. Then, a RT reaction system, including 5xRT buffer $(4.0 \mu \mathrm{l})$, RNA-free $\mathrm{H}_{2} \mathrm{O}(2.6 \mu \mathrm{l}), 10 \mathrm{mM}$ dNTPs (2.0 $\mu \mathrm{l}$; Promega, USA), RNasin $(0.4 \mu \mathrm{l})$ and M-MLV-RTase $(1.0 \mu \mathrm{l})$, was run for $1 \mathrm{~h}$ at $42^{\circ} \mathrm{C}$, then transferred to a $70^{\circ} \mathrm{C}$ water bath for $10 \mathrm{~min}$ to inactivate the RTase. The RT-qPCR cDNA product was placed at $-20^{\circ} \mathrm{C}$ for use. $\mathrm{PCR}$ was performed using $1.0 \mu \mathrm{l}$ cDNA with SYBRGreen PCR Master mix kit (Takara Bio, Inc., Otsu, Japan), according to the manufacturer's protocol. The RT-qPCR product was detected using an Agilent MX3000p qPCR system (Agilent Technologies, Inc., Santa Clara, CA, USA) with GAPDH as an internal control. The primer sequences were as follows: CENPU forward, 5'-ATGAACTGCTTCGGTTAGAGC-3' and reverse, 5'-TAT TTCGCAGATGGCTTTCGG-3'; and GAPDH forward, 5'-TGACTTCAACAGCGACACCCA-3' and reverse, 5'-CAC CCTGTTGCTGTAGCCAAA-3'. The PCR reaction was performed with an initial denaturation step of $95^{\circ} \mathrm{C}$ for $15 \mathrm{sec}$, followed by 45 cycles of $95^{\circ} \mathrm{C}$ for $5 \mathrm{sec}$ and $60^{\circ} \mathrm{C}$ for $30 \mathrm{sec}$. Following all the cycles, $95^{\circ} \mathrm{C}$ denaturation was performed for $1 \mathrm{~min}$, followed by cooling at $55^{\circ} \mathrm{C}$ for $30 \mathrm{sec}$. Next, from $55^{\circ} \mathrm{C}$, the temperature was increased in $0.5^{\circ} \mathrm{C}$ increments and maintained for $4 \mathrm{sec}$ at each step for absorbance readings until a final temperature of $95^{\circ} \mathrm{C}$ was achieved. The relative mRNA expression was calculated using the $2^{-\Delta \Delta C q}$ method (18), with GAPDH mRNA expression being used for normalization.

shRNA expression lentiviral vector construction and cell transfection. A short hairpin RNA targeting CENPU sequences (5'-CAGGTATGAGCTATAATAA-3') and a control with scrambled non-silencing RNA (5'-TTCTCCGAACGT GTCACGT-3') were designed and synthesized by Shanghai Genechem Co., Ltd., (Shanghai, China). The synthesized fragments at a final concentration of $100 \mathrm{M}$ were inserted into the lentiviral vector GV115 at the AgeI/EcoRI sites to form the recombinant lentiviral CENPU shRNA expression vector and 
Table I. CENPU expression in paired breast cancer and adjacent normal tissues.

\begin{tabular}{lcccc}
\hline Samples & N & CENPU expression (\%) & $\chi^{2}$ & P-value \\
\hline Breast cancer & 15 & $14(93.30)$ & 11.627 & 0.001 \\
Normal breast tissues & 15 & $5(33.30)$ & &
\end{tabular}

CENPU, centromere protein $U$.

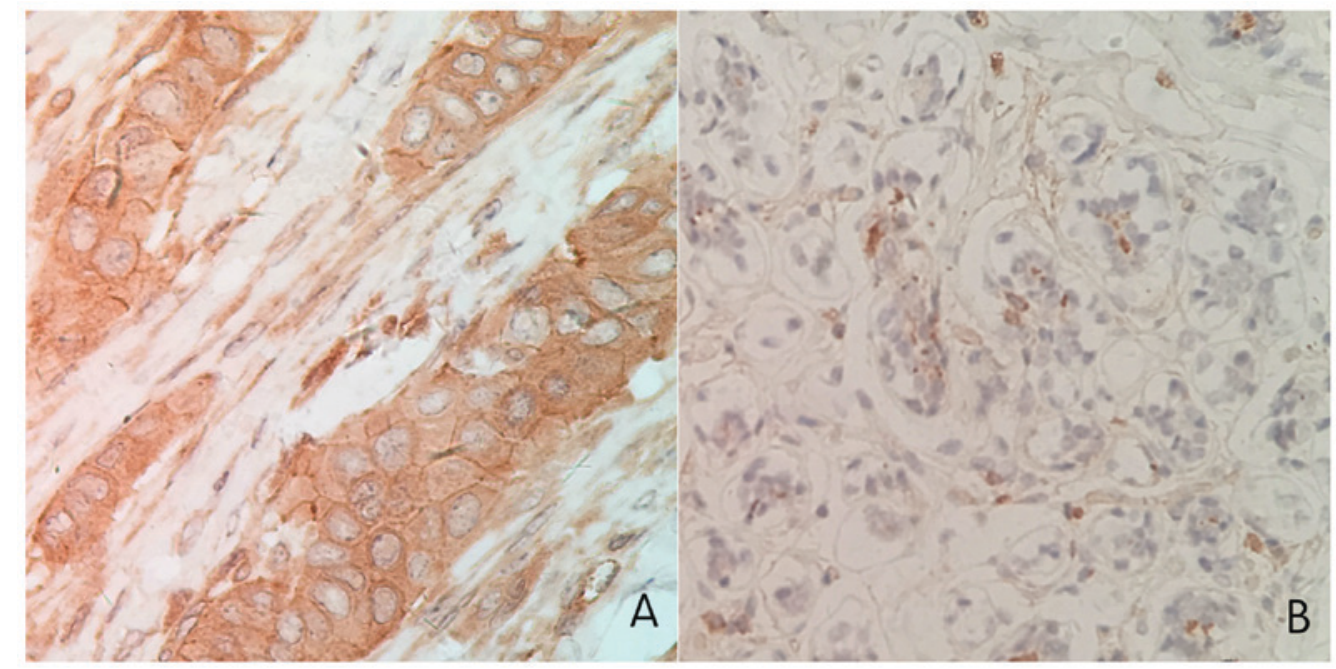

Figure 1. Immunohistochemical staining for CENPU. (A) Positive CENPU is immunostaining results in breast cancer tissue (magnification, $\mathrm{x} 400$ ). (B) Negative CENPU immunostaining results in normal tissues (magnification, x200). CENPU, centromere protein $\mathrm{U}$.

its control vector. The accuracy of the constructed vectors was verified via DNA sequencing. The verified lentiviral vectors carrying CENPU shRNA and non-silencing RNA were packaged by co-transfecting with Helper 1.0 and Helper 2.0 plasmids (Shanghai Genechem Co., Ltd., Shanghai, China) in 293(T) cells. The resulting recombinant lentiviruses were then harvested by centrifugation $\left(4^{\circ} \mathrm{C}, 10 \mathrm{~min}, 4,000 \mathrm{x} \mathrm{g}\right)$ and purification after 48 -h cell culture and designated shCENPU and shCtrl. For lentivirus transfection, the MDA-MB-231 cell culture in the logarithmic phase was treated with trypsin (0.25\%, pH=8.0, Shanghai Chemical Reagent Co., Ltd., Shanghai, China) at $37^{\circ} \mathrm{C}$ for $1-2 \mathrm{~min}$ and then re-suspended in DMEM (Gibco; Thermo Fisher Scientific, Inc.). The cell suspension $\left(3-5 \times 10^{4}\right.$ cells) was seeded onto a six-well plate and incubated at $37^{\circ} \mathrm{C}$ in $5 \% \mathrm{CO}_{2}$ until it reached $30 \%$ confluence. A total of $2 \times 10^{6} \mathrm{TU} / \mathrm{ml}$ lentivirus was then added to the wells according to the multiplicity of infection (obtained from a preliminary experiment). The transfection efficiency was measured by green fluorescent protein (GFP) fluorescence (Shanghai Genechem Co., Ltd., Shanghai, China), and CENPU gene expression of the transfected cells was evaluated using RT-qPCR for mRNA and western blotting for protein 3 days after the transfection.

Western blotting. The transfected shCENPU cells and shCtrl cells were harvested and lysed in 2X SDS Lysis buffer (Shanghai Chemical Reagent Co., Ltd.). The protein determination was performed following the BCA Protein assay kit (Shanghai Beyotime Biotech Co., Ltd.) Equal amounts of protein $(20 \mu \mathrm{g})$ were separated using 10\% SDS-PAGE and electrotransferred onto polyvinylidene difluoride membranes (EMD Millipore, Billerica, MA, USA), according to the standard operating procedure (19-21). The membranes were then blocked with $5 \%$ dry skimmed milk in Tris-buffered saline with Tween-20 (0.1\%) at room temperature for $1 \mathrm{~h}$ and incubated with a primary rabbit anti-human CENPU polyclonal antibody (dilution, 1:500; cat. no. ab117078; Abcam, Cambridge, UK) at $4^{\circ} \mathrm{C}$ overnight. A mouse anti-GAPDH monoclonal antibody (dilution, 1:2,000; cat. no. sc-32233; Santa Cruz Biotechnology, Inc., Dallas, TX, USA) was used as the loading control. Subsequent to washing with TBST (Shanghai Chemical Reagent Co., Ltd.) at room temperature for $8 \mathrm{~min}$ for 4 times, the membranes were incubated with a horseradish peroxidase-conjugated goat-anti rabbit or goat anti-mouse secondary antibody (dilution, 1:2,000; cat. no. sc-2004, 2005, Santa Cruz Biotechnology, Inc., Dallas, TX, USA) and visualized using an Enhanced Chemiluminesence Western Blotting Substrate kit (Pierce; Thermo Fisher Scientific, USA).

Cell proliferation assay. The transfected shCENPU and shCtrl cells were plated in five 96-well plates at equal cell densities $\left(2,000\right.$ cells $/ 100 \mu \mathrm{l}$ in each well) and cultured at $37^{\circ} \mathrm{C}$ with $5 \% \mathrm{CO}_{2}$ for $24 \mathrm{~h}$. The plates were then analyzed for GFP expression in each well using the Celigo Imaging Cytometer (Nexcelom Bioscience, Lawrence, MA, USA) over a 5-day period. Statistical data mapping and construction of cell proliferation curves were then performed on the dataset by 
Table II. Analysis of the expression of CENPU genes in breast cancer and adjacent normal tissues from The Cancer Genome Atlas data.

CENPU expression

\begin{tabular}{lcccccc}
\cline { 3 - 5 } Samples & $\mathrm{N}$ & Upregulated & Unchanged & Downregulated & P-value & FC \\
\hline Paired breast cancer and adjacent normal tissues & 106 & $89(84 \%)$ & $17(16 \%)$ & $0(0 \%)$ & $<0.001$ & 4.630
\end{tabular}

P-values were determined by the generalized linear model using the edgeR software package and $\mathrm{P}<0.05$ is considered to indicate a statistically significant difference. CENPU, centromere protein U; FC, fold-change. The FC value was calculated as the cancer sample count divided by the adjacent normal sample count. Sample-unchanged: The number of samples with no significant difference in the original data (defined as the FC value of cancer counts relative to adjacent normal sample counts) was between 0.5 and 2 (i.e., $0.5<\mathrm{FC}<2$ ). Sample-upregulated: The number of samples with raw data increased (the definition of upregulation is that the cancer sample counts is equal to or more than twice the counts of the adjacent normal sample, i.e., FC $\geq 2$ ). Sample-downregulated: The number of samples with raw data downregulated (downregulation is defined as cancer sample counts $\leq 50 \%$ of the adjacent normal sample counts, i.e., FC $\leq 0.5$ ).

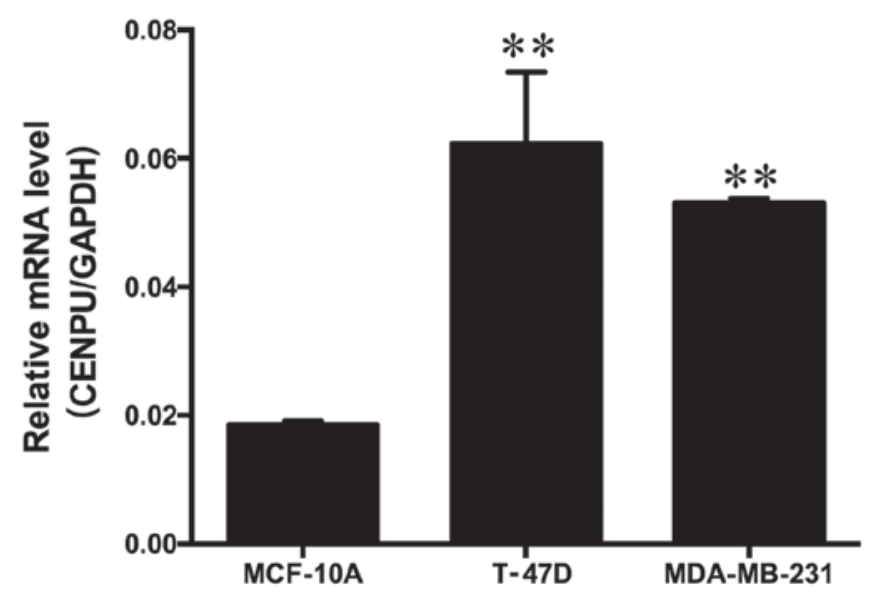

Figure 2. Reverse transcription-quantitative polymerase chain reaction results on CENPU mRNA expression. CENPU mRNA expression varied across the three cell lines, MCF-10A, T-47D and MDA-MB-231. GAPDH was used as an internal control. ${ }^{* *} \mathrm{P}<0.01$. CENPU, centromere protein $\mathrm{U}$.

GraphPad software (version 5.1, GraphPad Software, Inc., La Jolla, CA, USA).

An MTT assay was conducted using a commercial assay kit according to the manufacturer's protocol (GenView, Lausanne, Switzerland). In brief, the shCENPU and shCtrl transfected cell cultures were harvested from the primary cultures via trypsin treatment $\left(0.25 \%, 37^{\circ} \mathrm{C}, 1-2 \mathrm{~min}\right)$, followed by resuspension in DMEM (Gibco; Thermo Fisher Scientific, Inc.). The cells were seeded at equal density $(2,000$ cell/100 $\mu 1$ in each well) in five 96-well plates and incubated at $37^{\circ} \mathrm{C}$ in $5 \% \mathrm{CO}_{2}$ for continuous detection over a 5-day period. The first plate was analyzed after 24-h culture. The culture was terminated by adding $20 \mu \mathrm{l}$ MTT $(5 \mathrm{mg} / \mathrm{ml})$ to each well. After $4 \mathrm{~h}$ incubation, the medium was aspirated, and $100 \mu \mathrm{l}$ dimethyl sulfoxide (Shanghai Pharmaceutical Group Co., Ltd., Shanghai, China) was added to each well to dissolve the purple formazan. The plates were then oscillated for $5 \mathrm{~min}$ and the optical densities at $490 \mathrm{~nm}$ of each well were measured using a M2009PR microplate reader (Tecan Group, Ltd., Mannedorf, Switzerland).

Cell cycle assay. The shCENPU and shCtrl cell cultures were treated with trypsin and resuspended in DMEM (Gibco;
Thermo Fisher Scientific, Inc.) after achieving $80 \%$ confluence. Subsequent to washing with D-Hanks (Shanghai Genechem Co., Ltd.), the cells were counted by a hemocytometer to ensure a sufficient number of cells ( $\geq 1 \times 10^{6} /$ well with three wells per experimental group) were present and pelleted via centrifugation $(200 \mathrm{x} \mathrm{g})$ at room temperature for $5 \mathrm{~min}$. The cell pellet was washed in pre-cooled D-Hanks ( $\mathrm{pH}, 7.2-7.4)$, fixed in $70 \%$ ethanol at $4^{\circ} \mathrm{C}$ for $2-3 \mathrm{~h}$, and stained with $0.6-1 \mathrm{ml}$ propidium iodide solution [40x PI liquor ( $2 \mathrm{mg} / \mathrm{ml}$; cat. no. P4170; Sigma-Aldrich; Merck KGaA, Darmstadt, Germany), 100x RNase A liquor (10 mg/ml; cat. no. EN0531; Fermentas; Thermo Fisher Scientific, Inc., Pittsburgh, PA, USA), and 1x D-Hanks (Shanghai Genechem Co., Ltd.)]. The suspension was then filtered through a 300-mesh nylon mesh and analyzed by flow cytometry using a FACS Calibur instrument (EMD Millipore) at a flow rate of 300-800 cells/sec. Data analysis was performed using FlowJo Version 7.6.1 (FlowJo LLC, Ashland, OR, USA).

Apoptosis detection. The shCENPU and shCtrl cell cultures were treated with trypsin $\left(0.25 \%, 37^{\circ} \mathrm{C}, 1-2 \mathrm{~min}\right)$ and resuspended in the DMEM (Gibco; Thermo Fisher Scientific, Inc.) after reaching $85 \%$ confluence. Following washed with D-Hanks (Shanghai Chemical Reagent Co., Ltd.), the apoptosis status of the cell population was evaluated using the Annexin V-APC Apoptosis Detection kit (eBioscience; Thermo Fisher Scientific, Inc.) according to the manufacturer's protocol. In brief, cells were resuspended in binding buffer (Shanghai Chemical Reagent Co., Ltd., Shanghai, China) at a density of $5 \times 10^{5}$ cells $/ \mathrm{ml}$. A total of $200 \mu \mathrm{l}$ of this suspension was transferred to a $5 \mathrm{ml}$ tube with $10 \mu \mathrm{l}$ of the Annexin V-APC reagent added. Following gentle mixing, the cells were incubated at room temperature in dark for $15 \mathrm{~min}$. The cells were then analyzed by flow cytometry using a FACS Calibur instrument (EMD Millipore), Data analysis was performed using FlowJo Version 7.6.1 (FlowJo LLC, Ashland, OR, USA).

Statistical analysis. Statistical analysis was performed using SPSS software (version 19; IBM Corp., Armonk, NY, USA). Bar graphs represent the mean of the data from triplicate experiments with error bars indicating standard deviations (mean \pm standard deviation). Data analysis for multiple pairs of samples was performed using the generalized linear model 
A

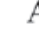

A

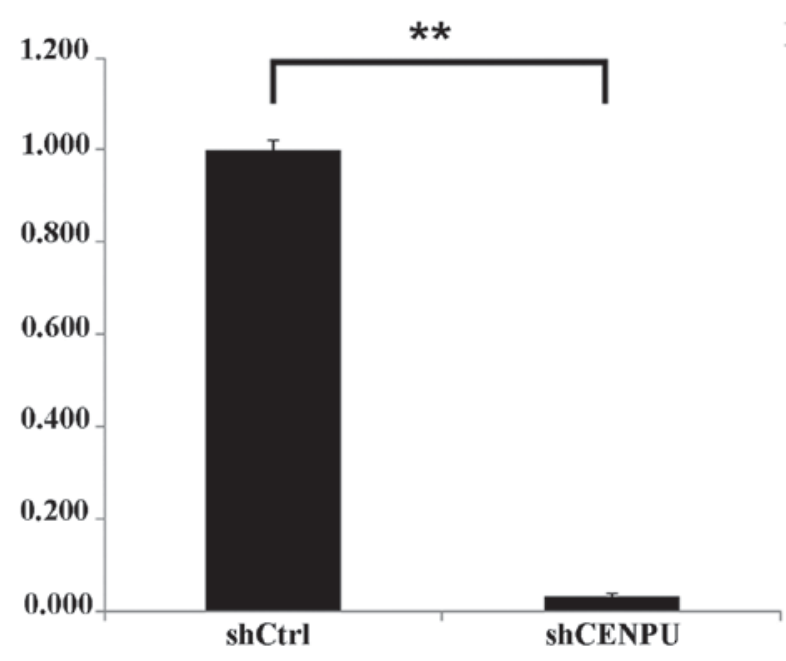

B

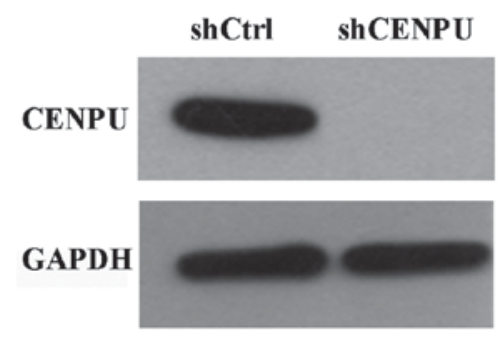

Figure 3. Reverse transcription-quantitative polymerase chain reaction and western blot analysis on CENPU expression in MDA-MB-231 cells following transfection. (A) Post-lentiviral transfection, relative CENPU mRNA expression was significantly inhibited in the shCENPU group compared with the shCtrl group. (B) The protein expression of CENPU was significantly downregulated in shCENPU relative to shCtrl. GAPDH was used as an internal control. ${ }_{* * *} \mathrm{P}<0.01$. CENPU, centromere protein U; shCENPU, CENPU-knockdown; shCtrl, negative control.

(GLM) using the edgeR software package (22). Comparisons between groups were performed using one-way analysis of variance and the $\chi^{2}$ test. $\mathrm{P}<0.05$ was considered to indicate a statistically significant difference.

\section{Results}

CENPU expression in breast cancer tissues, adjacent normal tissues and cell lines. CENPU protein expression was evaluated in 15 paired tumor and normal breast specimens via immunohistochemistry. The expression of CENPU was significantly increased in breast cancer tissues (93.3\%) than in adjacent normal tissues (33.3\%; Fig. 1; Table $\mathrm{I} ; \mathrm{P}=0.001$ ). Furthermore, a comprehensive analysis of a set of 106 breast cancer samples gene expression data from TCGA database revealed that CENPU mRNA was often upregulated in breast cancer tissues relative to non-tumor tissues, and CENPU mRNA expression was 4.63-fold higher in cancer tissues than in the normal tissues $(\mathrm{P}<0.001$; Table II). These results demonstrated that CENPU expression was significantly upregulated in breast cancer tissues compared with adjacent normal tissues.

The tissue expression results indicated that high expression of CENPU may also be present in the breast cancer cell lines. To test this hypothesis, the CENPU gene expression in two breast cancer cell lines (T-47D and MDA-MB-231) and the normal breast MCF-10A cell line was evaluated using RT-qPCR. It was identified that CENPU mRNA was highly expressed in breast cancer cell lines compared with the normal breast cell line (Fig. 2).

Knockdown of CENPU by lentivirus transfection in breast cancer cells. Since the MDA-MB-231 cell line represents a class of poorly differentiated, highly proliferative and aggressive breast cancer compared with T-47D, the MDA-MB-231 cell line was selected for the lentivirus-mediated CENPU-downregulation experiment to investigate the function of CENPU in cell proliferation and apoptosis. The shCENPU and shCtrl lentiviruses were generated and transfected into the MDA-MB-231 cell line. The lentivirus transfection efficiency was measured by GFP expression. After 3 days of transfection, $>80 \%$ of the cells were transfected. Post-transfection, the expression of CENPU in MDA-MB-231 cells was analyzed by RT-qPCR for mRNA expression and western blotting for protein expression. As presented in Fig. 3, the mRNA and protein expression levels of CENPU were significantly inhibited in CENPU-shRNA cells compared with the control cells $(\mathrm{P}<0.01)$, indicating successful downregulation of gene expression by CENPU-shRNA.

Downregulation of CENPU inhibits cell proliferation. The CENPU expression data presented earlier indicated that overexpression of the CENPU gene may be associated with enhanced proliferation of cancer cells. However, downregulation of CENPU expression inhibited cell proliferation. To test this hypothesis, the cell growth of the transfected breast cancer MDA-MB-231 cell line with the shCENPU and shCtrl lentiviruses was monitored by measuring the GFP expression and performing MTT assays over a 5 day period. As presented in Fig. 4, the proliferation of MDA-MB-231 cells was significantly decreased in the CENPU-shRNA cells compared with that of the control $(\mathrm{P}<0.01)$. The cell proliferation difference between the CENPU-shRNA cells and the control cells could be observed on the 2 nd day of analysis. The difference became more notable in a time-dependent manner, indicating the inhibitory effect of CENPU-knockdown on cell proliferation.

Downregulation of CENPU suppresses cell cycle progression. The effect of CENPU-downregulation on the cell cycle progression was further evaluated in breast cancer cells by FACS. The resulting data revealed that CENPU-downregulation induced a pronounced alteration in cell cycle distribution. After 5 days of lentivirus infection, the number of cells in the $\mathrm{G} 2+\mathrm{S}$ phase was significantly decreased in CENPU-knockdown cells relative to the control, whereas the number of cells in the G1 phase was significantly increased $(\mathrm{P}<0.01$; Fig. $5 \mathrm{~A})$. These data indicated that cells were arrested in the G1 phase following CENPU-downregulation. 

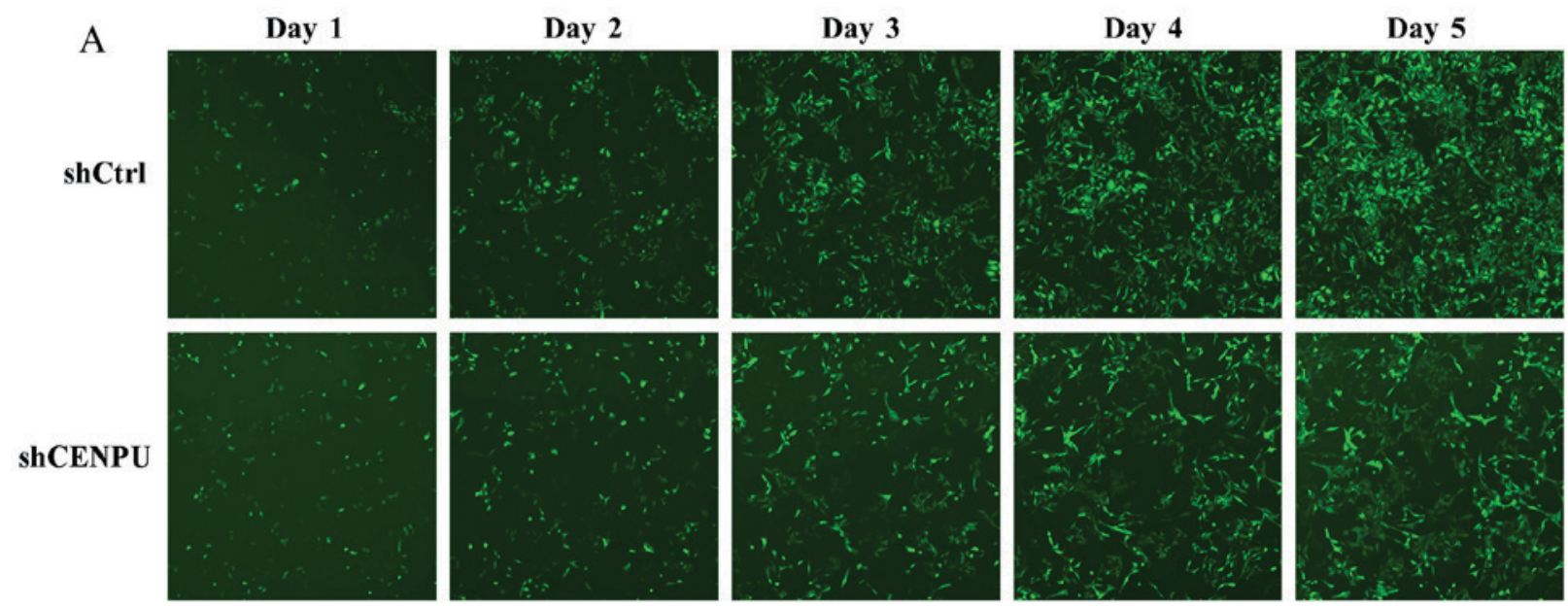

B
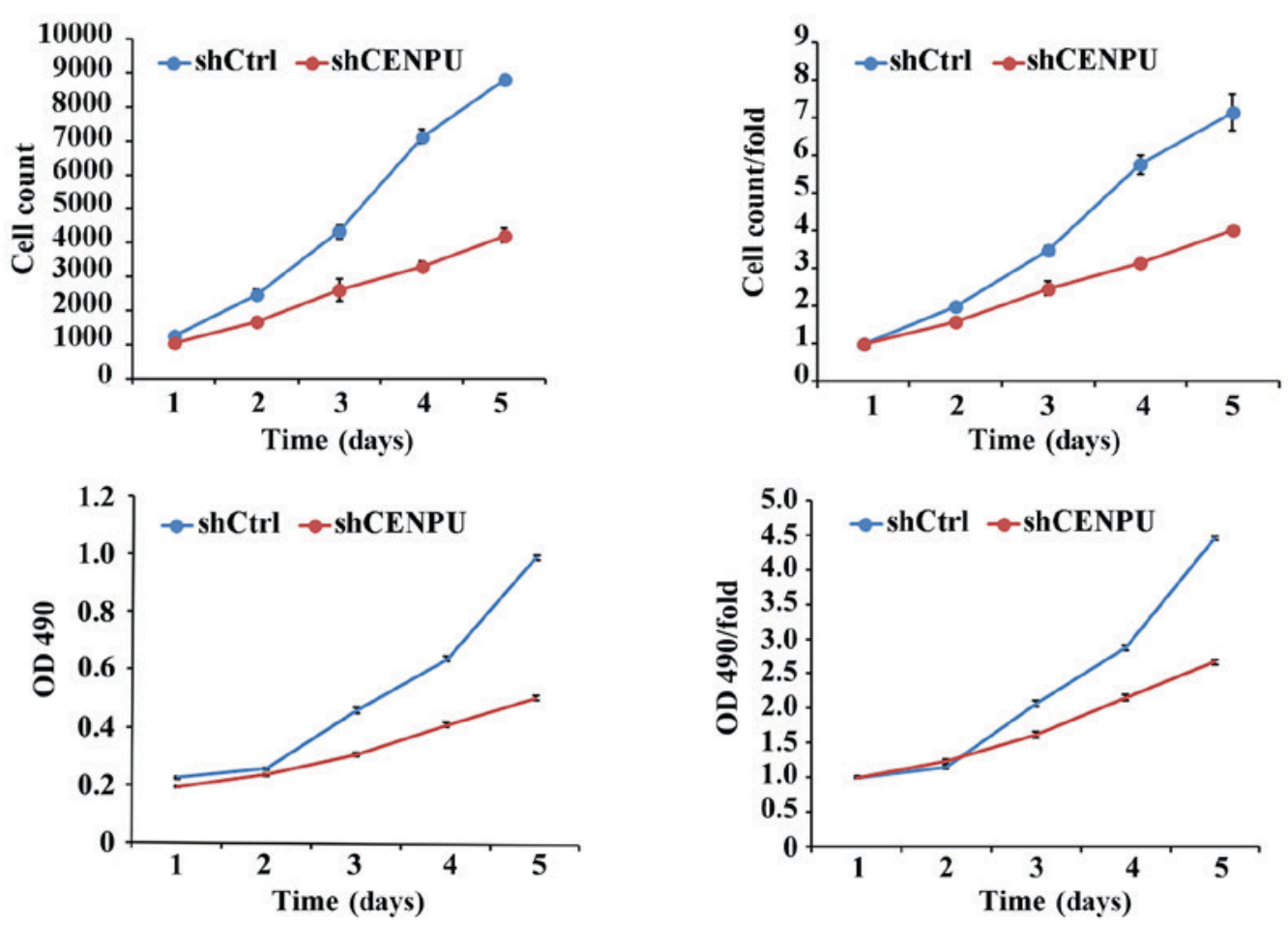

Figure 4. Cell proliferation analysis by GFP-based imaging and MTT assay. (A) Following lentiviral transfection of MDA-MB-231 cells, cell proliferation was significantly inhibited in shCENPU compared with shCtrl according to GFP-based Celigo imaging $(\mathrm{P}<0.01)$. (B) The MTT value ratio was significantly reduced in shCENPU compared with shCtrl $(\mathrm{P}<0.01)$. CENPU, centromere protein U; shCENPU, CENPU knockdown; shCtrl, negative control; GFP, green fluorescent protein.

Downregulation of CENPU gene expression promotes cell apoptosis. To investigate the effect of CENPU on cell apoptosis, Annexin V-APC staining and flow cytometric analysis were performed following lentivirus transfection. The data indicated that downregulation of CENPU gene expression in MDA-MB-231 cells was able to significantly increase the percentage of apoptotic cells compared with the percentage of control cells $(\mathrm{P}<0.01$; Fig. 5B), indicating a marked apoptotic effect of CENPU-downregulation.

\section{Discussion}

CENPU was initially identified as a novel protein that is specifically associated with Myeloid leukemia factor 1 (MLF1). It encodes a 46-kDa protein that contains two nuclear localization signals, two nuclear receptor binding motifs, two leucine zippers and several potential phosphorylation sites (13). The precise function of CENPU remains unresolved; however, activity as a suppressor of transcription has been demonstrated by a study using Kaposi's sarcoma-associated herpes virus (6). CENPU was also identified as a constitutive component of the centromere and served an important function in cell cycle progression $(23,24)$. It was required for maintenance of sister chromatid adhesion and stable kinetochore-microtubule attachment $(23,25)$. Deletion of CENPU may cause a $\mathrm{G}_{2} / \mathbf{M}$ delay and abnormal chromosome segregation (23). Previous studies revealed that CENPU was a phosphorylation substrate of PLK1, and that the phosphorylation-dependent CENPU-PLK1 interaction is required for PLK1 recruitment to the interphase and mitosis kinetochores, which are vital for proper mitotic progression $(24,26)$. 

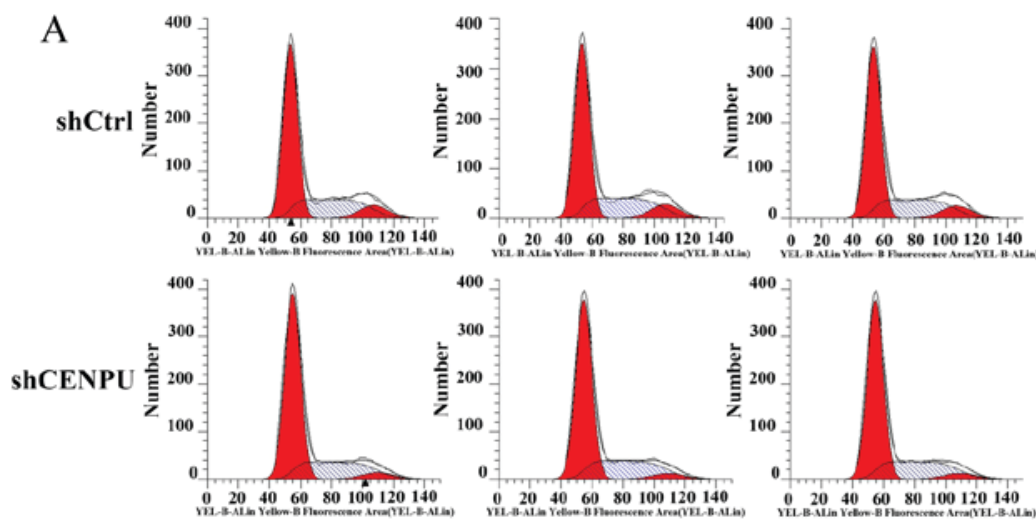

B
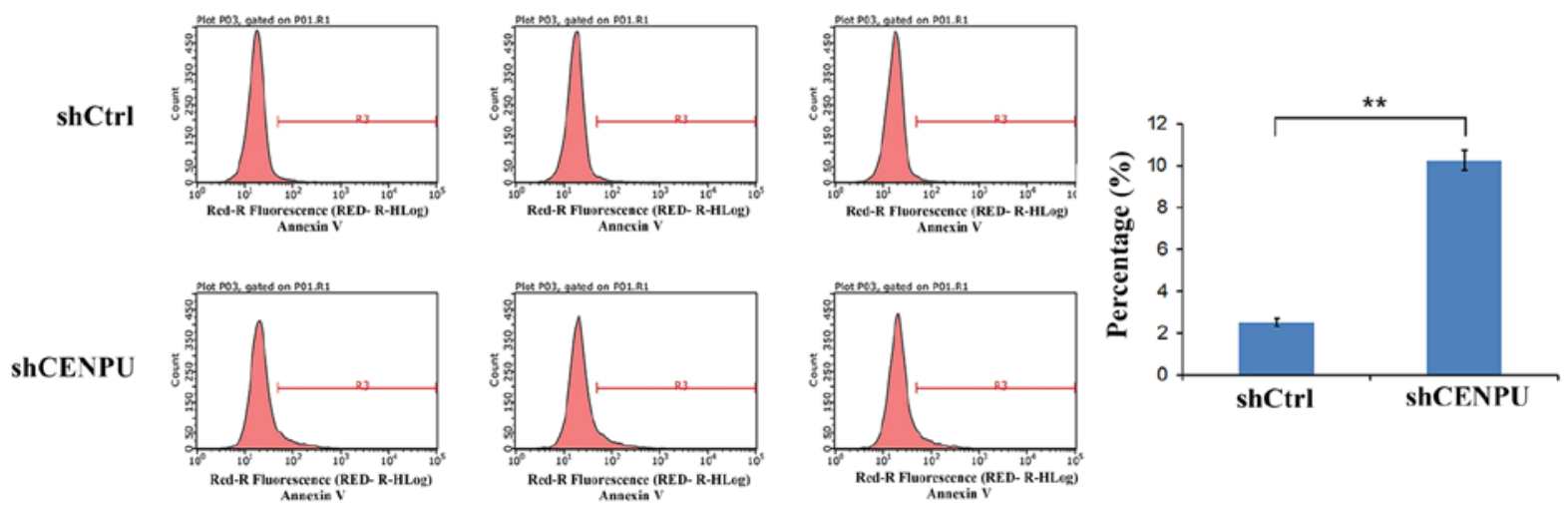

Figure 5. Cell cycle analysis by flow cytometry and Apoptotic activity by Annexin V-APC assay. (A) Cell cycle analysis by flow cytometry. Following lentiviral transfection of MDA-MB-231 cells, the cell counts in the G1, S and G2 phases were significantly altered in the shCENPU group compared with the shCtrl group. (B) Apoptotic activity by Annexin V-APC assay. Following lentiviral transfection of MDA-MB-231 cells, apoptosis was significantly increased in shCENPU compared with shCtrl. " $\mathrm{P}<0.01$. CENPU, centromere protein U; shCENPU, CENPU-knockdown; shCtrl, negative control.

The number of studies on the association between CENPU expression and human carcinoma is increasing. Hanissian et al $(11,13)$ have identified that CENPU, which was co-expressed with MLF1, may serve an important function in erythroleukemias and glioblastoma pathogenesis by preventing cell apoptosis and facilitating cell proliferation. Zhang et al (10) demonstrated that CENPU-knockdown was able to significantly suppress prostate cancer cell proliferation and colony formation, and promote cell apoptosis. In addition to these studies, Chou et al (27) have identified that CENPU is one of the genes most commonly associated with breast cancer by pooled cDNA microarray analysis using logistic regression, artificial neural networks and decision trees. Huang et al (12) demonstrated that CENPU is significantly upregulated in luminal breast cancer tissue in comparison with adjacent normal tissue in a validated cohort and a TCGA cohort. In the present study, immunohistochemical and TCGA analysis suggested that CENPU expression was significantly upregulated in human breast cancer. In addition, RT-qPCR analysis revealed that CENPU mRNA was also highly expressed in breast cancer T-47D and MDA-MB-231 cell lines compared with the normal breast cell line. These results suggested a possible function of CENPU in breast cancer pathogenesis.

In order to clarify the cellular mechanism of CENPU in breast cancer development, the expression of CENPU was downregulated and the effects on the proliferation, apoptosis and cell cycle progression of the MDA-MB-231 cells was evaluated. As was identified by RT-qPCR and western blotting analysis, the mRNA and protein expressions of CENPU were significantly decreased in CENPU-shRNA cells relative to the control $(\mathrm{P}<0.01)$, which indicated successful transfection and gene expression knockdown. Furthermore, the present study demonstrated that the proliferation activity was significantly suppressed in CENPU-knockdown cells relative to the control $(\mathrm{P}<0.01)$. The inhibited cell growth may be induced by the alteration of the cell cycle or cell apoptosis; therefore, FACS was performed for cell cycle and apoptosis analysis. It was identified that downregulation of CENPU induced significantly increased cell cycle arrest at the G1 phase $(\mathrm{P}<0.01)$. In addition to dysregulated cell cycle progression, it was also identified that the percentage of apoptotic cells was increased following CENPU-downregulation $(\mathrm{P}<0.01)$.

In conclusion, the present study was the first to demonstrate the effect of CENPU silencing by lentiviral mediated RNA interference on the proliferation and apoptosis of breast cancer. Suggesting that downregulation of CENPU gene expression may suppress cell proliferation, increase the apoptotic rate and alter cell cycle distribution in human breast cancer cells. These results implied that CENPU may serve an essential function in breast cancer pathogenesis and development. Although the present study clearly illustrated the possible molecular mechanism of the CENPU gene in breast cancer development promoting cell proliferation and inhibiting cell apoptosis, further studies are required to precisely characterize the cellular and molecular mechanisms of CENPU and its potential relevance to the clinical and pathological parameters for patients with breast cancer. 


\section{Acknowledgements}

Not applicable.

\section{Funding}

The present study was supported by the Zhejiang Provincial Public Welfare Technology Research Program (grant no. LGF18H160028), the Zhejiang Provincial Health Bureau Foundation (grant no. 2013KYA004), the National Natural Science Foundation of China (grant no. 81771520) and the innovation discipline of Zhejiang Province to Xujiao Chen.

\section{Availability of data and materials}

The datasets used and/or analyzed during the current study are available from the corresponding author on reasonable request.

\section{Authors' contributions}

FP and SL made substantial contributions to study conception and design, SL, YL, GM and XC were involved in acquisition of data, analysis and interpretation of data. SL drafted the manuscript and revised it critically for important intellectual content.

\section{Ethics approval and consent to participate}

Written informed consent was obtained from each of the patients, in compliance with the Declaration of Helsinki, and the present study was approved by the Medical Ethics Committee of Zhejiang Hospital (approval no. 2017-19K).

\section{Patient consent for publication}

All the patients have written informed consent for the publication of any associated data and accompanying images.

\section{Competing interests}

The authors declare that they have no competing interests.

\section{References}

1. Yang WT and Zhu XZ: The introduction of 2012 WHO classification of tumours of the breast. Zhonghua Bing Li Xue Za Zhi 42: 78-80, 2013 (In Chinese).

2. Kreiter E, Richardson A, Potter J and Yasui Y: Breast cancer: Trends in international incidence in men and women. $\mathrm{Br}$ J Cancer 110: 1891-1897, 2014.

3. Makki J, Myint O, Wynn AA, Samsudin AT and John DV: Expression distribution of cancer stem cells, epithelial to mesenchymal transition, and telomerase activity in breast cancer and their association with clinicopathologic characteristics. Clin Med Insights Pathol 8: 1-16, 2015.

4. Makki J: Diversity of breast carcinoma: Histological subtypes and clinical relevance. Clin Med Insights Pathol 8: 23-31, 2015.

5. 5 Morris LG and Chan TA: Therapeutic targeting of tumor suppressor genes. Cancer 121: 1357-1368, 2015.

6. Lee YT, Tan YJ and Oon CE: Molecular targeted therapy: Treating cancer with specificity. Eur J Pharmacol 834: 188-196, 2018.

7. Bode AM, Dong Z and Wang H: Cancer prevention and control: Alarming challenges in China. Natl Sci Rev 3: 117-127, 2016.
8. Chen W, Zheng R, Baade PD, Zhang S, Zeng H, Bray F, Jemal A, Yu XQ and He J: Cancer statistics in China, 2015. CA Cancer J Clin 66: 115-132, 2016.

9. Pan HY, Zhang YJ, Wang XP, Deng JH, Zhou FC and Gao SJ: Identification of a novel cellular transcriptional repressor interacting with the latent nuclear antigen of Kaposi's sarcoma-associated herpesvirus. J Virol 77: 9758-9768, 2003.

10. Zhang L, Ji G, Shao Y, Qiao S, Jing Y, Qin R, Sun H and Shao C: MLF1 interacting protein: A potential gene therapy target for human prostate cancer? Med Oncol 32: 454, 2015.

11. Hanissian SH, Teng B, Akbar U, Janjetovic Z, Zhou Q, Duntsch C and Robertson JH: Regulation of myeloid leukemia factor-1 interacting protein (MLF1IP) expression in glioblastoma. Brain Res 1047: 56-64, 2005.

12. Huang DP and Luo RC: MLF1IP is correlated with progression and prognosis in luminal breast cancer. Biochem Biophys Res Commun 477: 923-926, 2016.

13. Hanissian SH, Akbar U, Teng B, Janjetovic Z, Hoffmann A, Hitzler JK, Iscove N, Hamre K, Du X, Tong Y, et al: cDNA cloning and characterization of a novel gene encoding the MLF1-interacting protein MLF1IP. Oncogene 23: 3700-3707, 2004.

14. Grbesa I, Pajares MJ, Martinez-Terroba E, Agorreta J, Mikecin AM, Larráyoz M, Idoate MA, Gall-Troselj K, Pio R and Montuenga LM: Expression of sirtuin 1 and 2 is associated with poor prognosis in non-small cell lung cancer patients. PLoS One 10: e0124670, 2015.

15. Cha EJ, Noh SJ, Kwon KS, Kim CY, Park BH, Park HS, Lee H, Chung MJ, Kang MJ, Lee DG, et al: Expression of DBC1 and SIRT1 is associated with poor prognosis of gastric carcinoma. Clin Cancer Res 15: 4453-4459, 2009.

16. You TK, Kim KM, Noh SJ, Bae JS, Jang KY, Chung MJ, Moon WS, Kang MJ, Lee DG and Park HS: Expressions of E-cadherin, Cortactin and MMP-9 in Pseudoepitheliomatous hyperplasia and squamous cell carcinoma of the head and neck: Their relationships with clinicopathologic factors and prognostic implication. Korean J Pathol 46: 331-340, 2012.

17. Chandran UR, Medvedeva OP, Barmada MM, Blood PD, Chakka A, Luthra S, Ferreira A, Wong KF, Lee AV, Zhang Z, et al: TCGA expedition: A data acquisition and management system for TCGA data. PLoS One 11: e0165395, 2016.

18. Livak KJ and Schmittgen TD: Analysis of relative gene expression data using real-time quantitative PCR and the 2(-Delta Delta C(T)) method. Methods 25: 402-408, 2001.

19. Towbin H, Staehelin T and Gordon J: Electrophoretic transfer of proteins from polyacrylamide gels to nitrocellulose sheets: Procedure and some applications. Proc Natl Acad Sci USA 76: 4350-4354, 1979.

20. Burnette WN: 'Western blotting': Electrophoretic transfer of proteins from sodium dodecyl sulfate-polyacrylamide gels to unmodified nitrocellulose and radiographic detection with antibody and radioiodinated protein A. Anal Biochem 112: 195-203, 1981 .

21. Fuentes JM, Lompré AM, Møller JV, Falson P and le Maire M: Clean Western blots of membrane proteins after yeast heterologous expression following a shortened version of the method of Perini et al. Anal Biochem 285: 276-278, 2000.

22. Robinson MD, McCarthy DJ and Smyth GK: edgeR: A Bioconductor package for differential expression analysis of digital gene expression data. Bioinformatics 26: 139-140, 2010.

23. Minoshima Y, Hori T, Okada M, Kimura H, Haraguchi T, Hiraoka Y, Bao YC, Kawashima T, Kitamura T and Fukagawa T: The constitutive centromere component CENP-50 is required for recovery from spindle damage. Mol Cell Biol 25: 10315-10328, 2005.

24. Lee KS, Oh DY, Kang YH and Park JE: Self-regulated mechanism of Plk1 localization to kinetochores: Lessons from the Plk1-PBIP1 interaction. Cell Div 3: 4, 2008.

25. Hua S, Wang Z, Jiang K, Huang Y, Ward T, Zhao L, Dou Z and Yao X: CENP-U cooperates with Hecl to orchestrate kinetochore-microtubule attachment. J Biol Chem 286: 1627-1638, 2011.

26. Hori T, Okada M, Maenaka K and Fukagawa T: CENP-O class proteins form a stable complex and are required for proper kinetochore function. Mol Biol Cell 19: 843-854, 2008.

27. Chou HL, Yao CT, Su SL, Lee CY, Hu KY, Terng HJ, Shih YW, Chang YT, Lu YF, Chang CW, et al: Gene expression profiling of breast cancer survivability by pooled cDNA microarray analysis using logistic regression, artificial neural networks and decision trees. BMC Bioinformatics 14: 100, 2013. 